Pacific Journal of Mathematics

ON THE BEHAVIOR OF PINCHERLE BASIS FUNCTIONS 


\section{ON THE BEHAVIOR OF PINCHERLE BASIS FUNCTIONS}

\section{MAYNARD ARSOVE}

A basis $\left\{\alpha_{n}\right\}$ in the space of analytic functions on a disc $\{z:|z|<R\}$ is called a Pincherle basis if, for each $n(=0,1, \cdots)$, the Taylor expansion of $\alpha_{n}(z)$ has $z^{n}$ as its first nonvanishing term. The object of the present work is to examine such sequences to determine how behavior of the individual functions $\alpha_{n}$ is related to the property that $\left\{\alpha_{n}\right\}$ is a basis. Of particular interest are the zeros of the functions $\psi_{n}(\boldsymbol{z})=\alpha_{n}(\boldsymbol{z}) / \boldsymbol{z}^{n}$, and the case when each $\psi_{n}$ is a linear function vanishing at a corresponding point $z_{n}$ is studied in detail. There exist bases in which infinitely many of the $z_{n}$ coincide with some point in the disc, or in which the $z_{n}$ cluster at the origin. Nevertheless, the basis property can be correlated with various growth-rate conditions on $\left\{z_{n}\right\}$. For example, if the sequence $\left\{\left|z_{0} z_{1} \cdots z_{n-1}\right|^{1 / n}\right\}$ converges to some number $A$, then the condition $A \geqq R$ is necessary and sufficient for $\left\{\alpha_{n}\right\}$ to be a basis. This and similar results are derived by using the automorphism theorem and properties of entire functions of exponential type. Correlations of this sort fail to materialize, however, for general (nonlinear) $\psi_{n}$, and certain phenomena encountered in this case are illustrated by examples involving nowhere vanishing $\psi_{n}$.

Although a great deal is now known about bases in topological linear spaces (see e.g. J. Marti [9]), the setting of analytic function spaces remains one of the most fruitful. In such spaces, primary interest attaches to the polynomial bases and the Pincherle bases. The latter are closely linked with the fundamental basis

$$
\delta_{n}(z)=z^{n} \quad(n=0,1, \cdots),
$$

which leads to considerable simplification, but Pincherle bases still turn out to be vastly more complicated than $\left\{\delta_{n}\right\}$. This will be imply evident in our discussion of the correlation between the individual functions $\alpha_{n}$ and the basis property. Certain aspects of the problem, discussed in [1] and [2], will be drawn on as needed. The automorphism theorem (about which more will be said presently) remains our principal tool and permits us to avoid use of the elaborate theory of basic series, developed for polynomials by J. M. Whittaker [11] and extended by W. F. Newns [10].

Let us recall a few of the relevant concepts. With only minor changes, the notation and terminology of [3] will be used throughout. Thus, we take the underlying space as the Fréchet space $\mathscr{F}_{R}$ of all functions analytic on a fixed open disc $N_{R}(0)$ of radius $R(0<R \leqq+\infty)$ 
about the origin, the topology being that of uniform convergence on compact sets. As in (1.1), the sequences that enter here will be presumed to be indexed by $n=0,1, \cdots$. A sequence $\left\{\alpha_{n}\right\}$ of functions in $\mathscr{F}_{R}$ is said to be a basis in $\mathscr{F}_{R}$ provided every function $f$ in the space can be represented as

$$
f=\sum_{n=0}^{\infty} c_{n} \alpha_{n},
$$

where $\left\{c_{n}\right\}$ is a sequence of complex numbers uniquely determined by $f$ and the convergence is uniform on compact sets. Moreover, the basis is proper if and only if the series figuring in (1.2) converges for exactly those sequences $\left\{c_{n}\right\}$ which are coefficient sequences for Taylor expansions of functions in $\mathscr{F}_{R}$.

Proper bases are important because of their connection with automorphisms on the space. By an automorphism on $\mathscr{F}_{R}$ we mean a linear homeomorphic mapping of $\mathscr{F}_{R}$ onto itself. The connection with proper bases is embodied in the automorphism theorem derived in [3] (see Theorem 2, p. 241): if $A$ is an automorphism on $\mathscr{F}_{R}$, then $\left\{A \delta_{n}\right\}$ is a proper basis in $\mathscr{F}_{R}$ and, conversely, if $\left\{\alpha_{n}\right\}$ is a proper basis in $\mathscr{F}_{R}$, then there exists an automorphism $A$ on $\mathscr{F}_{R}$ such that $\alpha_{n}=$ $A \delta_{n}(n=0,1, \cdots)$.

When the functions $\alpha_{n}$ are of the special form

$$
\alpha_{n}(z)=z^{n} \psi_{n}(z) \quad(n=0,1, \cdots),
$$

where each $\psi_{n}$ is a function in $\mathscr{F}_{R}$ with $\psi_{n}(0)=1$, the sequence $\left\{\alpha_{n}\right\}$ will be called a Pincherle sequence. The Pincherle basis problem then consists of determining conditions under which a given Pincherle sequence forms a basis in the space. Sufficient conditions for $\left\{\alpha_{n}\right\}$ to be a proper Pincherle basis in the space $\mathscr{F}_{\infty}$ of entire functions have been discussed in [1]. In particular, $\left\{\alpha_{n}\right\}$ will be such a basis if either

$$
\psi_{n}(z)=e^{\varphi(z)} \quad(n=0,1, \cdots)
$$

for some entire function $\varphi$ vanishing at the origin or $\psi_{n} \rightarrow 1$ uniformly on compact sets. In each case the zeros (if any) of $\psi_{n}$ diverge to $\infty$ as $n \rightarrow \infty$, and it thus appears that the limiting behavior of these zeros may be significant in the Pincherle basis problem.

With this as motivation, we proceed to examine the connection between the zeros of the functions $\psi_{n}$ and the values of $R$ for which $\left\{\alpha_{n}\right\}$ is a basis in $\mathscr{F}_{R}$. The special case in which each $\psi_{n}$ is linear is given particular emphasis, since this furnishes some interesting positive results. For example, suppose that each $\psi_{n}$ vanishes at some corresponding point $z_{n}$. Then the condition 


$$
\liminf _{n \rightarrow \infty}\left|z_{0} z_{1} \cdots z_{n-1}\right|^{1 / n} \geqq R
$$

turns out to be necessary for $\left\{\alpha_{n}\right\}$ to be be a basis in $\mathscr{F}_{R}$. This condition is not, in general, a sufficient one, but a derivation based on the automorphism theorem shows that it becomes sufficient whenever the sequence $\left\{\left|z_{0} z_{1} \cdots z_{n-1}\right|^{1 / n}\right\}$ is known to be convergent. Alternative sufficient conditions are

$$
\liminf _{n \rightarrow \infty}\left|z_{n}\right| \geqq R
$$

(proof based on the theorem of Boas cited in [2]) and

$$
0<\lim _{n \rightarrow \infty} \frac{\left|z_{0} z_{1} \cdots z_{n-1}\right|^{1 / n}}{n^{p}}<+\infty
$$

for some positive number $p$ (proof based on properties of entire functions of exponential type).

In the general case (when the functions $\psi_{n}$ are not required to be linear) the most decisive results are in the form of counterexamples. Indeed, we show that there exist sequences $\left\{\alpha_{n}\right\}$ of the type (1.3) which are not bases in $\mathscr{F}_{R}$, despite the fact that each $\psi_{n}$ nowhere vanishes (these functions can even be chosen to be uniformly bounded on compact sets). Thus it is apparent that the limiting behavior of the zeros is not a key to the general solution of the Pincherle basis problem. We show also that, for nowhere vanishing $\psi_{n}$, it is possible for $\left\{\alpha_{n}\right\}$ to be a basis in $\mathscr{F}_{R}$ for some values of $R$ and not for others.

Further information is given relative to the general basis problem, including an extension of the theorem of Boas to sequences of the form

$$
\beta_{n}(z)=\alpha_{n}(z) \psi_{n}(z),
$$

where $\left\{\alpha_{n}\right\}$ is a proper Pincherle basis in $\mathscr{F}_{R}$ and $\left\{\psi_{n}\right\}$ is a sequence of functions in $\mathscr{F}_{R}$ satisfying $\psi_{n}(0)=1(n=0,1, \cdots)$. In particular, this yields simple extensions of the condition (1.5) when the functions $\psi_{n}$ are linear.

2. Common zeros of the basis functions. Throughout the discussion, use will be made of the norms $\|f\|_{r}$ defined in terms of the power series expansion

$$
f(z)=\sum_{n=0}^{\infty} a_{n} z^{n}
$$

of a function $f$ in $\mathscr{F}_{R}$ as

$$
\|f\|_{r}=\sum_{n=0}^{\infty}\left|a_{n}\right| r^{n} \quad(0<r<R) .
$$


In particular, we draw on the results of $\S 3$ of [3], it being noted without further comment that (2.2) of [3] allows us to replace $M_{r}\left(\alpha_{n}\right)$ in these results by $\left\|\alpha_{n}\right\|_{r}$.

Some elementary properties of expansions in Pincherle bases will now be collected for convenient reference.

Lemma 2.1. Let $\left\{\alpha_{n}\right\}$ be a Pincherle sequence and $f$ a function in $\mathscr{F}_{R}$ having the expansion

$$
f=\sum_{n=0}^{\infty} c_{n} \alpha_{n}
$$

Then

$$
\limsup _{n \rightarrow \infty}\left|c_{n}\right|^{1 / n} \leqq 1 / R
$$

and a necessary and sufficient condition for $f$ to have a zero of order $m$ at the origin is that $c_{m}$ be the first nonvanishing coefficient in the expansion.

Proof. Condition (2.1) is immediate from Lemma 2 of [3], since obviously $\left\|\alpha_{n}\right\|_{r} \geqq r^{n}(n=0,1, \cdots)$, and the final assertion is readily verified from the assumed Pincherle character of $\left\{\alpha_{n}\right\}$.

Lemma 2.1 provides the following information on common zeros of the functions $\psi_{n}$.

LEMma 2.2. If $\left\{\alpha_{n}\right\}$ is a basis in some subspace of $\mathscr{F}_{R}$ containing all $\delta_{n}$, then the functions $\psi_{n}$ cannot ultimately have a common zero on $N_{R}(0)$.

Proof. Suppose that for some point $z_{0}$ in $N_{R}(0)$ and some positive integer $m$ we have $\psi_{n}\left(z_{0}\right)=0$ for all $n \geqq m$. An application of Lemma 2.1 with $f(z)=z^{m}$ then yields

$$
z_{0}^{m}=c_{m} \alpha_{m}\left(z_{0}\right)+c_{m+1} \alpha_{m+1}\left(z_{0}\right)+\cdots=0,
$$

forcing $z_{0}=0$, and this contradiction to the basic condition $\psi_{n}(0)=1$ $(n=0,1, \cdots)$ completes the proof.

On the other hand, the theory developed in $\S 4$ of [1] can be applied to obtain a Pincherle basis $\left\{\alpha_{n}\right\}$ in which infinitely many of the functions $\psi_{n}$ vanish at the same point.

EXAmple 2.3. The sequence $\left\{\alpha_{n}\right\}$ defined by

$$
\alpha_{n}(z)=\left\{\begin{array}{l}
z^{n}(1-z) \text { for } n \text { even } \\
z^{n}\left[1-\frac{z}{(n+1)(n+2)}\right] \text { for } n \text { odd }
\end{array}\right.
$$


is a proper Pincherle basis in $\mathscr{F}_{\infty}$.

Proof. We start with the everywhere convergent power series

$$
\varphi(z)=1+z+\frac{z^{2}}{3 !}+\frac{z^{3}}{3 !}+\frac{z^{4}}{5 !}+\frac{z^{5}}{5 !}+\cdots,
$$

i.e.

$$
\varphi(z)=\sum_{n=0}^{\infty} t_{n} z^{n}
$$

with

$$
t_{k-1}=t_{k}=1 / k ! \quad(k=1,3,5, \cdots) .
$$

The entire function $\varphi$ here has order 1 , since, clearly,

$$
e^{R / 2} \leqq \max _{|z|=R}|\varphi(z)| \leqq e^{R}
$$

We note also that $\varphi$ is of exponential type, the type $\tau$ being given according to formula (2.2.12) of [5] as

$$
\tau=\lim _{n \rightarrow \infty}\left|n ! t_{n}\right|^{1 / n}=1 \text {. }
$$

Hence, by Theorem 7 of [1], the functions

$$
\alpha_{n}(z)=z^{n}\left[1-\left(t_{n+1} / t_{n}\right) z\right] \quad(n=0,1, \cdots),
$$

which are those appearing in the statement of the example, comprise a proper basis in $\mathscr{F}_{\infty}$.

3. Some aspects of the linear case. As Example 2.3 indicates, the Pincherle sequences $\left\{\alpha_{n}\right\}$ for which all $\psi_{n}$ are linear exhibit important features of the behavior of Pincherle bases. We shall therefore examine such sequences in more detail. Specifically, it will be assumed that each $\psi_{n}$ is a nonconstant linear function, so that

$$
\alpha_{n}(z)=z^{n}\left(1-\frac{z}{z_{n}}\right) \quad(n=0,1, \cdots),
$$

where $z_{n}$ is the zero of $\psi_{n}$.

A simple criterion is at hand for determining when the subspace spanned by $\left\{\alpha_{n}\right\}$ contains the fundamental basis functions $\delta_{n}$.

LEMma 3.1. Let $\mathscr{A}$ be the subspace of $\mathscr{F}_{R}$ spanned by the sequence $\left\{\alpha_{n}\right\}$ of (3.1). Then the following three conditions are mutually equivalent:

(1) $\mathscr{A}$ contains at least one of the functions $\delta_{n}(n=0,1, \cdots)$; 
(2) $\mathscr{A}$ contains all of the functions $\delta_{n}(n=0,1, \cdots)$;

(3) the zeros $z_{n}$ of the functions $\alpha_{n}$ satisfy (1.4).

Proof. To show that (1) implies (3), let us assume that $\delta_{m}$ belongs to $\mathscr{A}$ for some $m$. Then, by Lemma 2.1, we have

$$
\delta_{m}=\sum_{n=m}^{\infty} c_{n} \alpha_{n},
$$

i.e.

$$
z^{m}=c_{m} 2^{m}\left(1-\frac{z}{z_{m}}\right)+c_{m+1} z^{m+1}\left(1-\frac{z}{z_{m+1}}\right)+\cdots
$$

Dividing by $z^{m}$ and letting $z \rightarrow 0$ yields $c_{m}=1$ and thereby

$$
0=-\frac{z}{z_{m}}+c_{m+1} z\left(1-\frac{z}{z_{m+1}}\right)+\cdots \cdot
$$

This, in turn, yields $c_{m+1}=1 / z_{m}$. Proceeding inductively, we find that

$$
c_{n}=\frac{1}{z_{m} z_{m+1} \cdots z_{n-1}}
$$

and condition (1.4) then follows from (2.1). Thus, (1) implies (3).

Since trivially (2) implies (1), the demonstration will be completed by showing that (3) implies (2). From (3.3) it is evident that the partial sums $s_{k}$ in (3.2) are given by

$$
s_{k}(z)=z^{m}-\frac{z^{m+k}}{z_{m} z_{m+1} \cdots z_{m+k-1}} \quad(k=1,2, \cdots) .
$$

The assumed condition (1.4) gives rise to the inequality

$$
\left|z_{0} z_{1} \cdots z_{n-1}\right|>\rho^{n}
$$

for fixed $\rho(0<\rho<R)$ and large $n$, so that

$$
\limsup _{k \rightarrow \infty}\left|s_{k}(z)-z^{m}\right| \leqq \limsup _{k \rightarrow \infty}\left|z_{0} z_{1} \cdots z_{m-1}\right|(r / \rho)^{m+k}=0
$$

uniformly in $|z| \leqq r<\rho$. Inasmuch as this holds for each choice of $m$, it is clear that (3) implies (2).

Note that formula (3.3) for the coefficient $c_{n}$ can be generalized as follows (again, by a simple inductive argument). Let $f$ be a function analytic on some neighborhood of the origin. If the Taylor's series for $f$ is

$$
f(z)=\sum_{n=0}^{\infty} a_{n} z^{n}
$$


and if $f$ admits an expansion in terms of the functions $\alpha_{n}$ of (3.1) as

$$
f(z)=\sum_{n=0}^{\infty} c_{n} z^{n}\left(1-\frac{z}{z_{n}}\right),
$$

then

$$
c_{0}=a_{0} \text { and } c_{n}=a_{n}+\frac{c_{n-1}}{z_{n-1}} \quad(n \geqq 1) .
$$

An obvious consequence of Lemma 3.1 is that, when $\left\{\alpha_{n}\right\}$ is a basis in $\mathscr{F}_{R}$, then the zeros of the functions $\alpha_{n}$ must satisfy (1.4). On the other hand, a sequence $\left\{\alpha_{n}\right\}$ of the form (3.1) may fail to be a basis in $\mathscr{F}_{R}$ even though its zeros satisfy (1.4). That is, the dense subspace $\mathscr{A}$, which contains all $\delta_{n}$, may fail to be closed in $\mathscr{F}_{R}$. This possibility is illustrated by

EXAMPLE 3.2. The sequence $\left\{\alpha_{n}\right\}$ defined by

$$
\alpha_{n}(z)=\left\{\begin{array}{l}
z^{n}\left(1-2^{n} z\right) \text { for } n \text { even } \\
z^{n}\left(1-\frac{z}{2^{n+1}}\right) \text { for } n \text { odd }
\end{array}\right.
$$

is not a basis in any $\mathscr{F}_{R}(0<R<+\infty)$. However, for $R \leqq 1$, the subspace $\mathscr{A}$ of $\mathscr{F}_{R}$ spanned by $\left\{\alpha_{n}\right\}$ contains all $\delta_{n}$.

Proof. That $\mathscr{A}$ contains all $\delta_{n}$ is immediate from Lemma 3.1, since it is plain that

$$
\left|z_{0} z_{1} \cdots z_{n-1}\right|^{1 / n}=\left\{\begin{array}{l}
2 \text { for } n \text { even }(\geqq 2) \\
1 \text { for } n \text { odd }
\end{array}\right.
$$

Suppose now that $\left\{\alpha_{n}\right\}$ spans $\mathscr{F}_{R}$ for some $R(0<R<+\infty)$, and consider the function $f$ in $\mathscr{F}_{R}$ defined by

$$
f(z)=\sum_{n=0}^{\infty}\left(\frac{z}{R}\right)^{n}
$$

According to (3.4) the coefficients $c_{n}$ of the expansion of $f$ in the basis $\left\{\alpha_{n}\right\}$ satisfy

$$
c_{n}=\frac{1}{R^{n}}+\frac{c_{n-1}}{z_{n-1}} \geqq \frac{1}{R^{n}} \quad(n \geqq 1) .
$$

There follows

$$
c_{n} \geqq \frac{c_{n-1}}{z_{n-1}} \geqq \frac{2^{n-1}}{R^{n-1}}
$$


for odd $n \geqq 3$, so that

$$
\limsup _{n \rightarrow \infty}\left|c_{n}\right|^{1 / n} \geqq \frac{2}{R} .
$$

This contradiction to Lemma 2.1 proves that $\left\{\alpha_{n}\right\}$ cannot be a basis in $\mathscr{F}_{R}$.

The remainder of our discussion of the linear case hinges on the following application of the automorphism theorem.

THEOREM 3.3. Let $\left\{z_{n}\right\}$ be any sequence of complex numbers satisfying

$$
\liminf _{n \rightarrow \infty}\left|z_{0} z_{1} \cdots z_{n-1}\right|^{1 / n} \geqq R,
$$

and let $\varphi$ be the function in $\mathscr{F}_{R}$ defined by

$$
\varphi(z)=1+\sum_{n=1}^{\infty} \frac{z^{n}}{z_{0} z_{1} \cdots z_{n-1}} .
$$

Further, let

$$
\alpha_{n}(z)=z^{n}\left(1-\frac{z}{z_{n}}\right) \quad(n=0,1, \cdots),
$$

and let $\left\{\beta_{n}\right\}$ be defined in terms of the successive remainders in (3.6) as

$$
\left\{\begin{array}{l}
\beta_{0}(z)=\varphi(z), \\
\beta_{n}(z)=\left(z_{0} z_{1} \cdots z_{n-1}\right) \sum_{k=n}^{\infty} \frac{z^{k}}{z_{0} z_{1} \cdots z_{k-1}} \quad(n \geqq 1) .
\end{array}\right.
$$

Then $\left\{\alpha_{n}\right\}$ is a proper basis in $\mathscr{F}_{R}$ if and only if $\left\{\beta_{n}\right\}$ is a proper basis in $\mathscr{F}_{R}$.

Proof. We see at once that

$$
\delta_{n}=\beta_{n}-\frac{1}{z_{n}} \beta_{n+1} \quad(n=0,1, \cdots) .
$$

Hence, if $T$ is an automorphism on $\mathscr{F}_{R}$ such that $\beta_{n}=T \delta_{n}(n=0,1$, $\cdots)$, then $\alpha_{n}=T^{-1} \delta_{n}(n=0,1, \cdots)$. This proves that $\left\{\alpha_{n}\right\}$ is a proper basis in $\mathscr{F}_{R}$ whenever $\left\{\beta_{n}\right\}$ is. For the converse we assume that $\left\{\alpha_{n}\right\}$ is a proper basis in $\mathscr{F}_{R}$ and take $T^{-1}$ as an automorphism on $\mathscr{F}_{R}$ such that $\alpha_{n}=T^{-1} \delta_{n}(n=0,1, \cdots)$. There results

$$
T \delta_{n}=\delta_{n}+\frac{1}{z_{n}} T \delta_{n+1} \quad(n=0,1, \cdots),
$$

and a simple recursive argument leads to 


$$
T \delta_{0}=\delta_{0}+\sum_{k=1}^{n} \frac{\delta_{k}}{z_{0} z_{1} \cdots z_{k-1}}+\frac{T \delta_{n+1}}{z_{0} z_{1} \cdots z_{n}} \quad(n \geqq 1) .
$$

As noted in pp. 240-241 of [3], the continuity of $T$ ensures that to any given $r(0<r<R)$ there corresponds a $\rho(0<\rho<R)$ such that for some constant $K$

$$
\left\|T \delta_{n}\right\|_{r} \leqq K \rho^{n} \quad(n=0,1, \cdots) .
$$

Passing to the limit on $n$ in (3.10), we thus obtain

$$
T \delta_{0}=\delta_{0}+\sum_{k=1}^{\infty} \frac{\delta_{k}}{z_{0} z_{1} \cdots z_{k-1}}=\beta_{0} .
$$

By repeated application of (3.9), in the form $T \delta_{n+1}=z_{n}\left(T \delta_{n}-\delta_{n}\right)$, we infer that $\beta_{n}=T \delta_{n}(n=0,1, \cdots)$, and the automorphism theorem ensures that $\left\{\beta_{n}\right\}$ is a proper basis in $\mathscr{F}_{R}$. This completes the proof.

In the light of Theorem 3.3, we turn our attention now to the sequences $\left\{\beta_{n}\right\}$.

Lemma 3.4. Let $\left\{\beta_{n}\right\}$ be defined as in (3.7). If

$$
\limsup _{n \rightarrow \infty}\left(\left\|\beta_{n}\right\|_{r}\right)^{1 / n}<R
$$

for all $r<R$, then $\left\{\beta_{n}\right\}$ is a proper Pincherle basis in $\mathscr{F}_{R}$ and the coefficients in the expansion

$$
f=\sum_{n=0}^{\infty} c_{n} \beta_{n}
$$

are given in terms of the Taylor coefficients $a_{n}$ of $f$ as

$$
c_{0}=a_{0} \text { and } c_{n}=a_{n}-\frac{a_{n-1}}{z_{n-1}}
$$

Proof. Linear independence of $\left\{\beta_{n}\right\}$ is obvious from Lemma 2.1, and there remains only to show that $\left\{\beta_{n}\right\}$ spans $\mathscr{F}_{R}$ to conclude that $\left\{\beta_{n}\right\}$ is a basis in $\mathscr{F}_{R}$. By Lemma 1 of [3] we know that the hypothesis (3.11) ensures that $\sum c_{n} \beta_{n}$ converges uniformly on compact sets whenever $\left\{c_{n}\right\}$ is a sequence of complex numbers satisfying

$$
\limsup _{n \rightarrow \infty}\left|c_{n}\right|^{1 / n} \leqq 1 / R
$$

Moreover, (3.8) shows that any function $f$ in $\mathscr{F}_{R}$ can be expressed as

$$
f(z)=\sum_{n=0}^{\infty} a_{n} z^{n}=\sum_{n=0}^{\infty} a_{n}\left[\beta_{n}(z)-\frac{1}{z_{n}} \beta_{n+1}(z)\right] .
$$

Since $\sum\left|a_{n} \beta_{n}\right|$, and therefore $\sum\left|\left(a_{n} / z_{n}\right) \beta_{n+1}\right|$, converge uniformly on 
compact subsets of $N_{R}(0)$, we can regroup terms in the preceding expansion to obtain

$$
f(z)=a_{0} \beta_{0}(z)+\sum_{n=1}^{\infty}\left(a_{n}-\frac{a_{n-1}}{z_{n-1}}\right) \beta_{n}(z),
$$

the convergence again being uniform on compact subsets of $N_{R}(0)$. This proves that $\left\{\beta_{n}\right\}$ spans $\mathscr{F}_{R}$ and establishes the asserted interrelationship between the coefficients $c_{n}$ and $a_{n}$. Finally, we note that condition (3.11) forces the Pincherle basis $\left\{\beta_{n}\right\}$ to be proper, in view of Theorem 1 of [3].

Our applications of Lemma 3.4 make use of the following direct estimate for $\left\|\beta_{n}\right\|_{r}$ :

$$
\left\|\beta_{n}\right\|_{r} \leqq\left|z_{0} z_{1} \cdots z_{n-1}\right| \sum_{k=n}^{\infty} \frac{r^{k}}{\left|z_{0} z_{1} \cdots z_{k-1}\right|}
$$

when $0<r<R$ and $n \geqq 1$.

Let us suppose that the limit

$$
\lambda=\lim _{n \rightarrow \infty}\left|z_{0} z_{1} \cdots z_{n-1}\right|^{1 / n}
$$

exists finitely, with $\lambda \geqq R$. Then, given $r<R$ and choosing $\rho$ so that $r<\rho<\lambda$, we have for large $n$

$$
\left\|\beta_{n}\right\|_{r} \leqq r^{n} \frac{\left|z_{0} z_{1} \cdots z_{n-1}\right|}{\rho^{n}} \cdot \frac{\rho}{\rho-r} .
$$

There results

$$
\limsup _{n \rightarrow \infty}\left(\left\|\beta_{n}\right\|_{r}\right)^{1 / n} \leqq \frac{r \lambda}{\rho},
$$

and, in the limit as $\rho \rightarrow \lambda$, this yields

$$
\limsup _{n \rightarrow \infty}\left(\left\|\beta_{n}\right\|_{r}\right)^{1 / n} \leqq r \quad(0<r<R) .
$$

Hence, by Lemma 3.4, $\left\{\beta_{n}\right\}$ is a proper Pincherle basis in $\mathscr{F}_{R}$.

In conjunction with Lemma 3.1, the above considerations furnish a definitive solution of the basis problem for sequences (3.1) such that (3.13) exists finitely. We state the conclusions as

THEOREM 3.5. If $\left\{\left|z_{0} z_{1} \cdots z_{n-1}\right|^{1 / n}\right\}$ has a finite limit, then the condition

$$
\lim _{n \rightarrow \infty}\left|z_{0} z_{1} \cdots z_{n-1}\right|^{1 / n} \geqq R
$$

is necessary and sufficient for the sequence 


$$
\alpha_{n}(z)=z^{n}\left(1-\frac{z}{z_{n}}\right) \quad(n=0,1, \cdots)
$$

to form a basis in $\mathscr{F}_{R}$, and the resulting basis is proper.

In particular, whenever the sequence $\left\{\left|z_{n}\right|\right\}$ itself converges, the criterion (3.14) simplifies to

$$
\lim _{n \rightarrow \infty}\left|z_{n}\right| \geqq R \text {. }
$$

We note here that the condition

$$
\liminf _{n \rightarrow \infty}\left|z_{n}\right| \geqq R
$$

is sufficient for the sequence $\left\{\alpha_{n}\right\}$ of (3.15) to be a proper basis in $\mathscr{F}_{R}$. Indeed, this appears as a trivial special case of the following theorem of Boas: every Pincherle sequence

$$
\varphi_{n}(z)=z^{n}\left[1+\lambda_{n}(z)\right] \quad(n=0,1, \cdots)
$$

for which

$$
\limsup _{n \rightarrow \infty}\left\|\lambda_{n}\right\|_{r}<1
$$$$
(0<r<R)
$$

is a proper basis in $\mathscr{F}_{R}$ (see Theorem 4.1 of Boas [6], also [2] and pp. 377-378 of [4]). Although replacement of the limit in (3.16) by the corresponding limit inferior in (3.17) thus gives rise to a sufficient condition for $\left\{\alpha_{n}\right\}$ to be a basis, no such analogue holds in (3.14). Existence of the limit in (3.14) is essential to Theorem 3.5, as is apparent from Example 3.2.

4. Further results in the linear case. In spite of its generality, the criterion formulated in Theorem 3.5 has one important omission. It is not applicable to the space of entire functions, since $R$ must be finite. As a means of dealing with this case (and incidentally obtaining further information for the case of finite $R$ ), we introduce an auxiliary function $\Phi$ closely related to the function $\phi$ of (3.6).

For $\left\{z_{n}\right\}$ any sequence of complex numbers satisfying (3.5), let $\Phi$ be the function in $\mathscr{F}_{R}$ defined by

$$
\Phi(z)=1+\sum_{n=1}^{\infty} \frac{z^{n}}{\left|z_{0} z_{1} \cdots z_{n-1}\right|} \quad(|z|<R) .
$$

Then the usual formula for the remainder in a Taylor's expansion leads to the inequality 


$$
\left\|\beta_{n}\right\|_{r} \leqq\left|z_{0} z_{1} \cdots z_{n-1}\right| \frac{\Phi^{(n)}\left(r_{n}\right)}{n !} r^{n} \quad(r<R),
$$

where $\beta_{n}$ is any of the functions defined in (3.7) and $r_{n}$ is some corresponding number between 0 and $r$. The fact that $\Phi^{(n)}(t)$ is nondecreasing for $0<t<R$ allows us to conclude that

$$
\left\|\beta_{n}\right\|_{r} \leqq \frac{\Phi^{(n)}(r)}{\Phi^{(n)}(0)} r^{n} \quad(n=0,1, \cdots)
$$

for $0<r<R$.

Using the estimate (4.2) in Lemma 3.4, we arrive at

THEOREM 4.1. Let $\left\{z_{n}\right\}$ be a sequence of complex numbers satisfying

$$
\liminf _{n \rightarrow \infty}\left|z_{0} z_{1} \cdots z_{n-1}\right|^{1 / n} \geqq R,
$$

and let $\Phi$ be defined as in (4.1). Then the condition

$$
\limsup _{n \rightarrow \infty}\left[\frac{\Phi^{(n)}(r)}{\Phi^{(n)}(0)}\right]^{1 / n} \leqq 1
$$

is sufficient for the sequence

$$
\alpha_{n}(z)=z^{n}\left(1-\frac{z}{z_{n}}\right) \quad(n=0,1, \cdots)
$$

to form a proper basis in $\mathscr{F}_{R}$.

As an application, suppose that $\left\{z_{n}\right\}$ is a sequence of complex numbers such that, for some positive number $p$, the limit

$$
\lim _{n \rightarrow \infty} n^{-p}\left|z_{0} z_{1} \cdots z_{n-1}\right|^{1 / n}
$$

exists finitely and is different from zero. The same then holds for the limit

$$
\lim _{n \rightarrow \infty} n\left|z_{0} z_{1} \cdots z_{n-1}\right|^{-\rho / n}
$$

$\rho$ being taken as $1 / p$. This implies that $\Phi$ is an entire function of order $\rho$ and finite type $\tau$, with

$$
\lim _{n \rightarrow \infty}(n / e)^{1-\rho}\left|\Phi^{(n)}(z)\right|^{\rho / n}=\tau \rho
$$

for each point $z$ of the complex plane (see Boas [5], p. 11). The conditions of Theorem 4.1 are thus satisfied for all $R$, and we have proved

THEOREM 4.2. If $\left\{z_{n}\right\}$ is a sequence of complex numbers such that 
the limit

$$
\lim _{n \rightarrow \infty} \frac{\left|z_{0} z_{1} \cdots z_{n-1}\right|^{1 / n}}{n^{p}}
$$

exists as a nonzero real number for some positive number $p$, then

$$
\alpha_{n}(z)=z^{n}\left(1-\frac{z}{z_{n}}\right) \quad(n=0,1, \cdots)
$$

defines a proper basis in $\mathscr{F}_{R}$ for every $R(0<R \leqq+\infty)$.

A somewhat more general result holds in the space of entire functions. In fact, when $R=+\infty$, it is clear from the derivation of Theorem 4.1 that condition (4.3) there can be replaced by the weaker condition that its left-hand member be finite. This, in turn, permits a relaxation of the limit hypothesis (4.4) to yield

THEOREM 4.3. If $\left\{z_{n}\right\}$ is a sequence of complex numbers such that

$$
\liminf _{n \rightarrow \infty} \frac{\left|z_{0} z_{1} \cdots z_{n-1}\right|^{1 / n}}{n^{p}}>0
$$

and

$$
\limsup _{n \rightarrow \infty} \frac{\left|z_{0} z_{1} \cdots z_{n-1}\right|^{1 / n}}{n^{p}}<+\infty
$$

for some positive number $p$, then

$$
\alpha_{n}(z)=z^{n}\left(1-\frac{z}{z_{n}}\right) \quad(n=0,1, \cdots)
$$

defines a proper basis in $\mathscr{F}_{\infty}$.

We remark that Theorem 4.3 serves to generalize Theorem 7 of [1]. Moreover, the assertions in Example 2.3 follow at once from either Theorem 4.2 or Theorem 4.3, so that it is clear that these theorems have some direct applicability. Our next example, however, is based on a slightly different approach, contained in the concluding paragraph of $\$ 4$ of [1].

EXAMPLE 4.4. There exists a basis of the form (3.1) in $\mathscr{F}_{\infty}$ for which the zeros $z_{n}$ cluster at the origin. In fact, the sequence

$$
\alpha_{n}(z)=\left\{\begin{array}{l}
z^{n}\left(1-\frac{2^{n+1}}{n+1} z\right) \text { for } n \text { even } \\
z^{n}\left(1-\frac{1}{(n+1) 2^{n}} z\right) \text { for } n \text { odd }
\end{array}\right.
$$


defines a proper basis in $\mathscr{F}_{\infty}$.

This example shows that the sufficient condition (3.17) is not a necessary one. It also serves to illustrate the fact that a Pincherle basis in $\mathscr{F}_{R}$ need not be bounded on any neighborhood of the origin.

5. The general case. Although one might hope to relate the general case of Pincherle bases in some natural way to the linear case, we shall see that serious difficulties arise. Indeed, some rather curious phenomena occur when the functions $\psi_{n}$ of (1.3) are assumed to have no zeros at all, and these make it plain that the distribution of the zeros of the functions $\psi_{n}$ does not furnish a key to the general solution of the Pincherle basis problem. We thus turn our attention to some of the situations at hand for nowhere vanishing $\psi_{n}(n=0$, $1, \cdots)$.

ExAMPLE 5.1. The Pincherle sequence

$$
\alpha_{n}(z)=z^{n} e^{n z} \quad(n=0,1, \cdots)
$$

is not a basis in any $\mathscr{F}_{R}$ with $R>1$. In fact, for $R>1$, the subspace Af of $\mathscr{F}_{R}$ spanned by $\left\{\alpha_{n}\right\}$ contains none of the functions $\delta_{n}(n \geqq 1)$.

Proof. Writing (5.1) as $\alpha_{n}(z)=\left(z e^{z}\right)^{n}$, we see that every function $f$ in $\mathscr{A}$ has the form

$$
f(z)=F\left(z e^{z}\right),
$$

where $F$ is some function determined by $f$. Since the coefficient condition (2.1) guarantees that $F$ is in $\mathscr{F}_{R}$, we can differentiate (5.2) to obtain

$$
f^{\prime}(z)=(z+1) e^{z} F^{\prime}\left(z e^{z}\right)
$$

It follows that $\mathscr{A}$ consists exclusively of functions whose derivatives vanish at $z=-1$, and hence that none of the functions $\delta_{n}(n \geqq 1)$ belongs to $\mathscr{A}$.

In the above example, the sequence of functions $\psi_{n}(z)=e^{n z}$ is unbounded on every neighborhood of the origin. The following example avoids this feature.

Example 5.2. The Pincherle sequence

$$
\alpha_{n}(z)=\left\{\begin{array}{l}
z^{n} e^{z} \text { for } n \text { even } \\
z^{n} e^{-z} \text { for } n \text { odd }
\end{array}\right.
$$

defines a basis in $\mathscr{F}_{R}$ if and only if $R \leqq \pi / 4$. 
Proof. Fixing $R>0$, we examine the subspace $\mathscr{A}$ of $\mathscr{F}_{R}$ spanned by $\left\{\alpha_{n}\right\}$. To begin with, it is evident that $\mathscr{A}$ consists of exactly those functions $f$ which can be expressed as

$$
f(z)=e^{z} p(z)+e^{-z} q(z) \quad(|z|<R),
$$

where $p$ is an even function in $\mathscr{F}_{R}$ and $q$ is an odd function in $\mathscr{F}_{R}$. This results in

$$
f(-z)=e^{-z} p(z)-e^{z} q(z) \quad(|z|<R),
$$

so that the functions $f$ comprising $\mathscr{A}$ are precisely those functions in $\mathscr{F}_{R}$ satisfying the relationships

$$
\left\{\begin{array}{l}
e^{z} f(z)+e^{-z} f(-z)=\left(e^{2 z}+e^{-2 z}\right) p(z) \\
e^{-z} f(z)-e^{z} f(-z)=\left(e^{2 z}+e^{-2 z}\right) q(z)
\end{array}\right.
$$

for $|z|<R$.

The coefficient $e^{2 z}+e^{-2 z}$ appearing in the right-hand members of (5.6) vanishes at $z=\pi i / 4$ but at no $z$ of smaller modulus. Thus, if $R \leqq \pi / 4$, we can solve for $p$ and $q$ in terms of an arbitrarily given $f$ in $\mathscr{F}_{R}$. The resulting functions $p$ and $q$ are even and odd functions, respectively, for which (5.5) holds, and it is immediate that $\mathscr{A}=\mathscr{F}_{R}$ in this case.

On the other hand, if $R>\pi / 4$, we can choose $z=\pi i / 4$ in (5.6), so that the right-hand members both reduce to zero. The only even or odd functions $f$ in $\mathscr{A}$ are then seen to be those having zeros at $\pi i / 4$. Since $\mathscr{A}$ therefore contains none of the functions $\delta_{n}$, it is plain that $\left\{\alpha_{n}\right\}$ does not span $\mathscr{F}_{R}$ in this case.

It is of interest to note here the following theorem of Kraplin [8]. Let $\psi$ be a function analytic on $N_{1}(0)$ with Taylor expansion

$$
\psi(z)=1+\sum_{k=1}^{\infty} b_{k} z^{k},
$$

let $M=\sup \left|b_{k}\right|(k \geqq 1)$, and let $\left\{a_{n}\right\}$ be any bounded sequence of complex numbers. Then

$$
\alpha_{n}(z)=z^{n} \psi\left(a_{n} z\right) \quad(n=0,1, \cdots)
$$

defines a proper basis in $\mathscr{F}_{R}$ for $R=1 /[q(1+M)]$, where $q=\sup \left|a_{n}\right|$. As applied to Example 5.2, however, this yields only the weaker result that the sequence $\left\{\alpha_{n}\right\}$ there is a basis in $\mathscr{F}_{R}$ for $R=1 / 2$.

This clearly shows that the results of the linear case do not carry over directly to the general case. Nevertheless, these results can be put to use by drawing on procedures for constructing new bases from known ones. The Paley-Wiener theorem developed in [4] yield such procedures, and we call attention here to other methods. 
A scheme for combining two proper bases to form a new one is an easy outgrowth of the automorphism theorem.

Lemma 5.3. Suppose that $\left\{\alpha_{n}\right\}$ is a basis in $\mathscr{F}_{R}$ and that the functions $\alpha_{n}$ have Taylor expansions of the form

$$
\alpha_{n}(z)=\sum_{k=0}^{\infty} A_{n k} z^{k} \quad(n=0,1, \cdots) .
$$

If $\left\{\beta_{n}\right\}$ is a proper basis in $\mathscr{F}_{R}$, then the expansions

$$
\gamma_{n}=\sum_{k=0}^{\infty} A_{n k} \beta_{k} \quad(n=0,1, \cdots)
$$

define $\left\{\gamma_{n}\right\}$ as a basis in $\mathscr{F}_{R}$. Moreover, $\left\{\gamma_{n}\right\}$ is proper if $\left\{\alpha_{n}\right\}$ is.

Proof. Convergence of the expansions in (5.8) is an evident consequence of the assumption that the basis $\left\{\beta_{n}\right\}$ is proper. This hypothesis also yields an automorphism $T$ on $\mathscr{F}_{R}$ such that $\beta_{n}=$ $T \delta_{n}(n=0,1, \cdots)$. The lemma then follows by applying $T$ to $(5.7)$ and setting $\gamma_{n}=T \alpha_{n}(n=0,1, \cdots)$.

In particular, Lemma 5.3 can be utilized to synthesize proper Pincherle bases, starting from the linear case. That is, if the sequences

$$
\alpha_{n}(z)=z^{n}\left(1-\frac{z}{a_{n}}\right) \quad(n=0,1, \cdots)
$$

and

$$
\beta_{n}(z)=z^{n}\left(1-\frac{z}{b_{n}}\right) \quad(n=0,1, \cdots)
$$

define proper bases in $\mathscr{F}_{R}$, then so does the sequence

$$
\gamma_{n}(z)=z^{n}\left[1-\left(\frac{1}{a_{n}}+\frac{1}{b_{n}}\right) z+\frac{1}{a_{n} b_{n+1}} z^{2}\right] \text {. }
$$

Proceeding inductively, we obtain a general method for the synthesis of proper Pincherle bases of the form (1.3) in which the $\psi_{n}$ are polynomials of arbitrarily prescribed common degree.

Another method for generating new Pincherle bases from known ones hinges on theorems of Paley-Wiener type, similar to the theorem of Boas cited in $\S 3$. Theorem 9 of [4] and the corresponding theorem of Evgrafov [7, p. 117, Theorem 2] fall into this category. Here is a further variation on this theme. 


$$
\alpha_{n}(z)=z^{n} \psi_{n}(z) \quad(n=0,1, \cdots)
$$

define a Pincherle basis in $\mathscr{F}_{R}$, and let

$$
\beta_{n}(z)=\alpha_{n}(z)\left[1+\lambda_{n}(z)\right] \quad(n=0,1, \cdots),
$$

where each $\lambda_{n}$ is a function in $\mathscr{F}_{R}$ satisfying $\lambda_{n}(0)=0$. If

$$
\limsup _{n \rightarrow \infty}\left\|\psi_{n} \lambda_{n}\right\|_{r}<1
$$

for all $r<R$, then $\left\{\beta_{n}\right\}$ is a Pincherle basis in $\mathscr{F}_{R}$ and there exists an automorphism $A$ on $\mathscr{F}_{R}$ such that $\beta_{n}=A \alpha_{n}(n=0,1, \cdots)$.

Proof. Reasoning similar to that of [2] will be used, but certain modifications are essential. We now put

$$
T f(z)=\sum_{n=0}^{\infty} c_{n} \alpha_{n}(z) \lambda_{n}(z),
$$

where

$$
f(z)=\sum_{n=0}^{\infty} c_{n} \alpha_{n}(z)
$$

Convergence of the series for $T f$ is guaranteed by (5.9), and we have, in fact,

$$
\|T f\|_{r} \leqq \sum_{n=0}^{\infty}\left|c_{n}\right| r^{n}\left\|\psi_{n} \lambda_{n}\right\|_{r} \quad(r<R) .
$$

It is evident from Lemma 2.1 that if $f$ has a zero of order $m$ or greater at the origin, then

$$
\|T f\|_{r} \leqq\left(\sup _{n \geq m}\left\|\psi_{n} \lambda_{n}\right\|_{r}\right)\|f\|_{r} \quad(r<R) .
$$

This result can be applied directly to $T^{m} f$, since the condition $\lambda_{n}(0)=$ 0 assures us that $T^{m} f$ has a zero of order greater than or equal to $m$ at 0 .

Fixing $r$, we choose a corresponding value of $\delta<1$ such that

$$
\limsup _{n \rightarrow \infty}\left\|\psi_{n} \lambda_{n}\right\|_{r}<\delta
$$

It is then easy to see that there exist constants $m_{0}$ and $K$ (depending on $r$ ) such that

$$
\left\|T^{m} f\right\|_{r} \leqq K\|f\|_{r} \delta^{m}
$$

for $m>m_{0}$ and all $f$ in $\mathscr{F}_{R}$. This ensures convergence of the operator series 


$$
U=\sum_{n=0}^{\infty}(-T)^{n}
$$

The resulting linear operator $U$ is obviously continuous and has the property that $U=(I+T)^{-1}$, where $I$ is the identity operator. It follows that the operator $A=I+T\left(=U^{-1}\right)$ is an automorphism on $\mathscr{F}_{R}$ carrying $\alpha_{n}$ into $\beta_{n}(n=0,1, \cdots)$, and this forces $\left\{\beta_{n}\right\}$ to be a basis in $\mathscr{F}_{R}$.

6. Concluding remarks. Certain Pincherle sequences $\left\{\alpha_{n}\right\}$ have the following property: for some number $R_{0} \geqq 0,\left\{\alpha_{n}\right\}$ is a basis in $\mathscr{F}_{R}$ if $R<R_{0}$, but is not a basis in $\mathscr{F}_{R}$ if $R>R_{0}$. Example 5.2 illustrates this situation with $R_{0}=\pi / 4$, and further illustrations are immediate from Theorems 3.5 and 5.4. Such $R_{0}$, when it exists, is an analogue of the radius of convergence of a power series, and it will be referred to as the basis radius determined by $\left\{\alpha_{n}\right\}$. (Note also Newns [10], pp. 450-451.)

Not every Pincherle sequence admits a basis radius. We show, in fact, that a Pincherle sequence can be a proper basis in $\mathscr{F}_{\infty}$ but fail to be a basis in $\mathscr{F}_{R}$ for all finite values of $R$.

EXAMPle 6.1. The sequence $\left\{\alpha_{n}\right\}$ defined by

$$
\begin{aligned}
& \alpha_{0}(z)=1-z, \\
& \alpha_{n}(z)=\left\{\begin{array}{l}
z^{n}\left[1-\frac{2^{n} n^{n}}{(n+1)^{n+1}} z\right] \text { for } n(>0) \text { even } \\
z^{n}\left[1-\frac{n^{n}}{2^{n+1}(n+1)^{n+1}} z\right] \text { for } n \text { odd }
\end{array}\right.
\end{aligned}
$$

is a proper basis in $\mathscr{F}_{\infty}$ but is not a basis in $\mathscr{F}_{R}$ for $R$ finite.

Proof. Here $\left\{\alpha_{n}\right\}$ is a sequence of the form (3.1) in which the numbers $z_{n}(n=0,1, \cdots)$ are chosen so as to make

$$
\liminf _{n \rightarrow \infty} \frac{\left|z_{0} z_{1} \cdots z_{n-1}\right|^{1 / n}}{n}=1
$$

and

$$
\limsup _{n \rightarrow \infty} \frac{\left|z_{0} z_{1} \cdots z_{n-1}\right|^{1 / n}}{n}=2 .
$$

Thus, according to Theorem 4.3, $\left\{\alpha_{n}\right\}$ is a proper basis in $\mathscr{F}_{\infty}$. To prove that $\left\{\alpha_{n}\right\}$ cannot be a basis in $\mathscr{F}_{R}$ if $R$ is finite, let us assume the contrary. Then the function $f$ in $\mathscr{F}_{R}$ defined by 


$$
f(z)=\sum_{n=0}^{\infty}\left(\frac{z}{R}\right)^{n}
$$

admits an expansion in the basis $\left\{\alpha_{n}\right\}$ of the form $f=\sum c_{n} \alpha_{n}$. As in Example 3.2 there results $c_{n} \geqq 1 / R^{n}(n=1,2, \cdots)$, so that for odd $n \geqq 3$

$$
c_{n} \geqq \frac{c_{n-1}}{z_{n-1}} \geqq \frac{2^{n-1}(n-1)^{n-1}}{n^{n} R^{n-1}} .
$$

This forces

$$
\lim \sup _{n \rightarrow \infty}\left|c_{n}\right|^{1 / n} \geqq \frac{2}{R},
$$

contradicting (2.1), and our proof is complete.

It would be of interest to find a general characterization of those Pincherle sequences which admit a basis radius, but this remains an open question.

\section{REFERENCES}

1. M. G. Arsove, Proper Pincherle bases in the space of entire functions, Quart. J. Math., (Oxford) (2), 9 (1958), 40-54.

2. The Pincherle basis problem and a theorem of Boas, Math. Scand., 5 (1957), 271-275.

3. - Proper bases and linear homeomorphisms in spaces of analytic functions, Math. Ann., 135 (1958), 235-243.

4. - The Paley-Wiener theorem in metric linear spaces, Pacific J. Math., 10 (1960), 365-379.

5. R. P. Boas, Jr., Entire Functions, New York, 1954.

6. - Expansions of analytic functions, Trans. Amer. Math. Soc., 48 (1940), 467-487.

7. M. A. Evgrafov, The method of approximating systems in the space of analytic functions and its applications to interpolation, Trudy Moskovskovo Mat. Obshchestva, 5 (1956), 89-201.

8. M. A. Kraplin, Two examples of matrix methods in the study of quasi-power bases, Sbornik Nauchno-Tech. Raboti Azovo-Chernomorsk. Inst. Mech., 10 (1957), 313-322.

9. J. Marti, Introduction to the Theory of Bases, New York, 1969.

10. W. F. Newns, On the representation of analytic functions by infinite series, Philos. Trans. Royal Soc. London, 245 (1953), 429-468.

11. J. M. Whittaker, Sur les séries de base de polynomes quelconques, Paris, 1949.

Received July 24, 1971. Research supported in part by the National Science Foundation. 



\section{PACIFIC JOURNAL OF MATHEMATICS}

\section{EDITORS}

H. SAMELSON

Stanford University

Stanford, California 94305

C. R. Hовву

University of Washington

Seattle, Washington 98105
J. DugundJI

Department of Mathematics University of Southern California

Los Angeles, California 90007

RICHARD ARENS

University of California

Los Angeles, California 90024

\section{ASSOCIATE EDITORS}

E. F. BECKENBACH

B. H. NeumanN

F. WOLF

K. YoSHIDA

\section{SUPPORTING INSTITUTIONS}

UNIVERSITY OF BRITISH COLUMBIA

CALIFORNIA INSTITUTE OF TECHNOLOGY

UNIVERSITY OF CALIFORNIA

MONTANA STATE UNIVERSITY

UNIVERSITY OF NEVADA

NEW MEXICO STATE UNIVERSITY

OREGON STATE UNIVERSITY

UNIVERSITY OF OREGON

OSAKA UNIVERSITY
UNIVERSITY OF SOUTHERN CALIFORNIA

STANFORD UNIVERSITY

UNIVERSITY OF TOKYO

UNIVERSITY OF UTAH

WASHINGTON STATE UNIVERSITY

UNIVERSITY OF WASHINGTON

AMERICAN MATHEMATICAL SOCIETY

NAVAL WEAPONS CENTER

Printed in Japan by International Academic Printing Co., Ltd., Tokyo, Japan 


\section{Pacific Journal of Mathematics}

\section{Vol. 44, No. $1 \quad$ May, 1973}

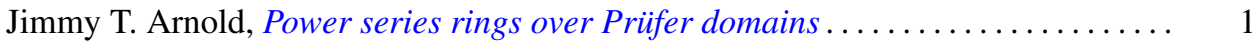

Maynard G. Arsove, On the behavior of Pincherle basis functions . . . . . . . . . 13

Jan William Auer, Fiber integration in smooth bundles ................. 33

George Bachman, Edward Beckenstein and Lawrence Narici, Function algebras

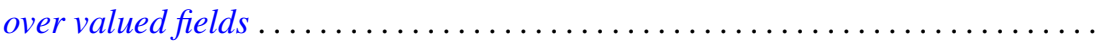

Gerald A. Beer, The index of convexity and the visibility function . . . . . . . . . . .

James Robert Boone, A note on mesocompact and sequentially mesocompact

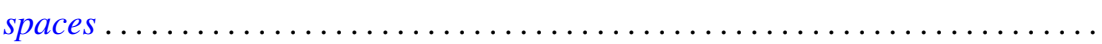

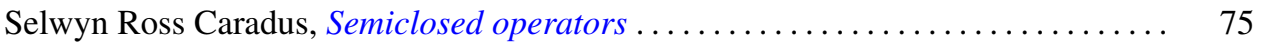

John H. E. Cohn, Two primary factor inequalities . . . . . . . . . . . . . . . 81

Mani Gagrat and Somashekhar Amrith Naimpally, Proximity approach to

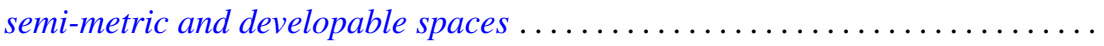

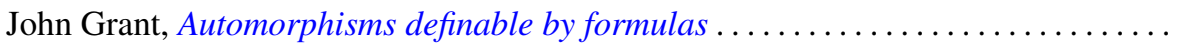

Walter Kurt Hayman, Differential inequalities and local valency ..............

Wolfgang H. Heil, Testing 3-manifolds for projective planes . . . . . . . . . . . . .

107

Melvin Hochster and Louis Jackson Ratliff, Jr., Five theorems on Macaulay

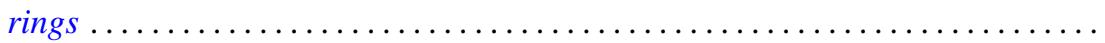

Thomas Benton Hoover, Operator algebras with reducing invariant subspaces ....

James Edgar Keesling, Topological groups whose underlying spaces are separable

Fréchet manifolds...

Frank Leroy Knowles, Idempotents in the boundary of a Lie group . .

191

George Edward Lang, The evaluation map and EHP sequences ...

201

Everette Lee May, Jr, Localizing the spectrum . . . . . . . . . . . .

211

Frank Belsley Miles, Existence of special $K$-sets in certain locally compact abelian groups.

Susan Montgomery, A generalization of a theorem of Jacobson. II . .

T. S. Motzkin and J. L. Walsh, Equilibrium of inverse-distance forces in

three-dimensions.

Arunava Mukherjea and Nicolas A. Tserpes, Invariant measures and the converse

of Haar's theorem on semitopological semigroups .

James Waring Noonan, On close-to-convex functions of order $\beta$

Donald Steven Passman, The Jacobian of a growth transformation

Dean Blackburn Priest, A mean Stieltjes type integral ........ .

Joe Bill Rhodes, Decomposition of semilattices with applications to topological

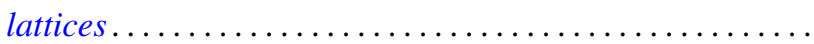

Claus M. Ringel, Socle conditions for $\mathrm{QF}-1$ rings ..........

Richard Rochberg, Linear maps of the disk algebra

Roy W. Ryden, Groups of arithmetic functions under Dirichlet convolution . .

Michael J. Sharpe, A class of operators on excessive functions

Erling Stormer, Automorphisms and equivalence in von Neumann algebras ..

Philip C. Tonne, Matrix representations for linear transformations on series

analytic in the unit disc. 